\title{
Perception of Conventional Medicine and Herbal Medicine Usage Amongst Diabetic Patients: A Qualitative Study in Negeri Sembilan, Malaysia
}

\author{
Norbaidurah Ithnain ${ }^{1}$, Albeny Joslyn Panting ${ }^{1}$, Rosnani Kassim ${ }^{1}$, Nadia Amirudin ${ }^{1} \&$ Manimaran Krishnan ${ }^{1}$ \\ ${ }^{1}$ Institute for Health Behavioural Research, Ministry of Health, Malaysia \\ Correspondence: Norbaidurah Ithnain, Institute for Health Behavioural Research, Ministry of Health Malaysia, \\ Block B3, National Institutes of Health Complex, No 1, Jalan Setia Murni U13/52, Section U13 Setia Alam, 40170 \\ Shah Alam, Selangor, Malaysia. Tel: 6-03-33-627-600.E-mail: durrah@moh.gov,my
}

Received: April 9, 2020 Accepted: July 7, 2020 Online Published: August 19, 2020

doi:10.5539/gjhs.v12n10p122 URL: https://doi.org/10.5539/gjhs.v12n10p122

\begin{abstract}
Introduction: The concomitant intake of herbal medicine and conventional medicine amongst patients is steadily increasing worldwide. However, concerns have been raised regarding potential adverse effects and drug interactions when consuming both medications together. Therefore, this work aimed to comprehending the perception of Type 2 Diabetic Mellitus (T2DM) patients on conventional medicine and the manner of its use with herbal medicine in managing diabetes mellitus.

Methods: A qualitative study was conducted among 28 diabetic patients in four government clinics under the jurisdiction of the state of Negeri Sembilan, Malaysia. Purposive sampling was employed to recruit informants who consumed herbal medicine alongside their prescribed conventional medicine. This study employed semi-structured interviews, which were all digitally recorded, transcribed, and analysed thematically.

Results: This study found that most of the patients perceived the negative side effects of conventional medicine, which led them to consider incorporating herbal medicine in treating diabetes. Such perception was influenced by the personal experiences of their family members and friends. A majority of the informants utilised herbal medicine as a complementary consumption to conventional medicine as opposed to implementing it as an alternative. However, they would alter the dosage and intake time of conventional medicine for several reasons, such as to complement their herbal medicine intake, being fed-up with conventional medicine, and reduce the risk of its side effects. Lastly, this study revealed that some of the patients noted their intention to discontinue conventional medicine, while some even tried to quit.

Conclusions: A complete understanding of patient's perceptions regarding conventional medicine and its usage with herbal medicine will aid healthcare providers to deliver education on the importance of conventional medicine usage compliance. Additionally, people should be aware that its combination with herbal medicine when consumed may present adverse effects and subsequently cause serious health problems.
\end{abstract}

Keywords: conventional medicine, herbal medicine, diabetes, patients

\section{Introduction}

Globally, diabetes prevalence has increased in recent years. In 2019, 163 million adults (20-79 years old) were living with the disease and 1.3 million deaths were recorded due to it in the International Diabetes Federation (IDF) Western Pacific Region. By 2045, the rates are projected to increase up to 212 million (IDF, 2019), whereby Malaysia is one of the 22 nations and localities under the umbrella of IDF Western Pacific Region. In the context of Malaysia, a study by the National Health Morbidity Survey logged over 3.492 .600 cases of diabetes in 2017 in which almost one in five Malaysian adults would have the disease (Ministry of Health, 2015).

It is now common knowledge that type 2 diabetes mellitus (T2DM) patients are taking conventional medicine prescribed as their treatment regiment, namely insulin and/or oral agents such as sulfonylureas, biguanides, meglitinides, alpha-glycosidase inhibitors, thiazolidinediones, and incretin mimetics (Ozkum et al., 2013). In particular, metformin has been accepted as the initial oral agent of choice and is widely used in diabetes management (Miller et al., 2009; Rojas \& Gomes, 2013). According to Rojas and Gomes, (2013), it is not only 
limited to glycaemic controls; metformin further offers improved effects endothelial dysfunction, haemostasis and oxidative stress, insulin resistance, lipid profile, and fat redistribution.

Although the use of conventional medicine is considered one of the vital avenues in diabetes, T2DM patients often seek alternatives in order to manage their disease, rendering herbal medicine as the current choice of treatment. Over the years, an increasing number has been recorded on herbal medicine usage amongst T2DM patients, especially in developing countries (Appiah et al., 2018). Its definition by the World Health Organisation (WHO) is as follows: herbs, herbal materials, herbal preparations, and finished herbal products containing parts of plants or other plant materials as the active ingredients (WHO, 2005). It is estimated that $80 \%$ of the global population implements herbal medicine as the principal health care formulation (WHO, 2002). Furthermore, more than 400 plant species are incorporated across various forms to reap their hypoglycaemic impacts for diabetes treatment purposes, in conjunction with sky-high appraisal of their effectiveness by patients and practitioners alike (Malviya et al., 2013).

Past research has found that most patients use herbal medicine to complement their conventional medicine prescription (Mwangi \& Gitonga, 2014; Zayneb et al., 2015; Vidya \& Lohit, 2019). According to Toklu (2016), a few reasons can be highlighted as the reason why it has been chosen by most people, such as their belief that herbal means natural, is safe, and poses fewer side effects than synthetic drugs. Furthermore, the National Centre for Complementary and Integrative Health (NCCIH 2016) has stated that complementary medicine is considered the integration of a non-mainstream practice with conventional medicine, including herbal medicine. Due to herbal medicine utilisation, some nations including China have incorporated both herbal and conventional medicines into their healthcare system (WHO, 2013).

In Malaysia, herbal medicine is defined as crude plant preparations used in various alternative medicine practices, including Malay medicine, traditional Malay medicine, Ayurvedic medicine, naturopathy, and homeopathy. Some are also marketed as nutritional food or dietary supplements. However, not much research has been undertaken regarding the use of local herbs as diabetes treatment or management (Ibrahim, 2006). According to Hasan et al., (2011), approximately half of the diabetic patients at Hospital Tuanku Jaa'far, Seremban was incorporating herbal medicine in order to complement their conventional treatment regimen. This outcome is in tandem with Remli and Chan's (2003) study undertaken at Hospital Ipoh, Perak, whereby it is noted that more than half of the diabetic patients complement their conventional medicine with herbal medicine. The reason for such usage is due to easy accessibility and its impact on the treatment of diabetes. In 2016, Baharom and colleagues conducted a cross-sectional study among T2DM patients in Negeri Sembilan, revealing that herbal medicine such as bitter gourd was chosen to help them manage their diabetes.

Despite the increasing use of herbal medicine intake amongst T2DM patients, it is important for one to understand the manner in which they perceive the conventional medicine and use it with herbal medicine in managing diabetes. To date, limited qualitative research is available and explored these matters in more depth and broader range. According to Mays et al., (2000), qualitative research methodologies are proven as highly useful in the field of healthcare, as they generate detailed understanding regarding the perceptions, beliefs, and values of the individuals and groups in question.

\section{Methods}

\subsection{Study Design and Setting}

This qualitative study was conducted to explore the perception of T2DM patients on conventional medicine and the manner in which they utilised it with herbal medicine for managing diabetes. The study sample consisted of T2DM patients hailing from four government clinics in Negeri Sembilan, Malaysia. This study was conducted in four government clinics in Negeri Sembilan, namely Klinik Kesihatan Seremban, Klinik Kesihatan Port Dickson, Klinik Kesihatan Kuala Pilah, and Klinik Kesihatan Bahau. Their selection was based on the Report on Diabetic Patient's Information (2016), whereby these four clinics yielded the highest number of diabetes registration.

An in-depth interview (IDI) was carried out with informants sampled via purposive sampling. The purposive sampling method is highly effectual in cases where a limited amount of individuals may serve as the principal data sources, which is attributable to the research design and research aims and objectives. This method requires the use of personal judgment in order to select cases for the purpose of answering the research questions or achieving the research objectives.

\subsection{Interview Process}

The informants were selected according to their eligibility, which included: aged 18 years and above, diagnosed with diabetes for more than three years, and incorporated herbal medicine alongside the conventional medicine. 
Their participation was confirmed for an interview as arranged by the staff nurse at the respective selected clinics. The interviews required 40-60 minutes to be completed by the moderator, while a research assistant was tasked to take notes. Each session was conducted in closed rooms for the convenience of the interview. First, the interview was initiated by a brief introduction on the objective of the study and the informants were asked to answer the consent forms. The IDIs were digitally recorded with the permission of informants, whereby the interview process was eased by an interview guide or interview protocol developed to facilitate it. To gain maximum data from the informants, the researcher used mainly open-ended questions and incorporated the probing technique.

\subsection{Analysis}

A tape recorder was utilised to record the interview sessions and their contents were transcribed ad verbatim. By recording the interviews, the researcher was afforded a high level of precision that would be otherwise not obtained from the field notes. However, this study further implemented the field notes to record any non-verbal communication, which would be highly valuable during data interpretation. The analysis process started by listening to the recordings and reading the transcribed content line by line in order to identify and extract data according to the initial codes relevant to the study objective. Then, codes that were similar to each other were applied to all transcripts and the resulting theme/subthemes were identified. These themes might be identifiable according to previous literature, while new themes might arise spontaneously from the informants during the interviews.

\subsection{Ethical Considerations}

Ethical approval was obtained from the Medical Research Ethics Committee Ministry of Health (NMRR ID: 17-953-35849) and the approval for undertaking the study was granted at the State and Clinic Levels. The informants were informed of the anticipated advantages of this work, while also reassured of their confidentiality and anonymity. Furthermore, their freedom to withdraw at any point of time was guaranteed and further verbal permission was taken from each of them in order to consent to audio-recording the interviews. After verifying their willingness for involvement, the informants completed the informed consent form and returned it to the researcher.

\section{Results}

\subsection{Overview of Informants}

A total of 28 IDI was conducted with diabetic patients, which consisted of 13 female and 15 male patients with an average age of 58 years. All informants had been diagnosed and undertook treatment with their respective health clinics for more than three years in four government clinics in Negeri Sembilan, Malaysia. The informants comprised of three different ethnicities, namely Malay, Chinese, and Indian.

\subsection{Conventional Medicine and Herbal Medicine Use}

Firstly, 13 informants were prescribed with both metformin and insulin in managing their diabetes, while five used metformin and gliclazide. One of them was consuming three medications concurrently, namely metformin, diamicron, and gliclazide, whereas the remaining were prescribed with insulin, metformin, diamicron, and gliclazide. In terms of herbal medicine usage, the informants described their herbal intake as primarily herbal medicine in different forms. Some informants said that it included raw local herbs, such as Momordica Charantia (bitter gourd), Trigonella Foenum-Graecum (fenugreek), Orthosiphon Stamineus (Cat's Whiskers), and Clinacanthus Nutans (Sabah Snake Grass). Meanwhile, others informed that it included natural plants such as pennywort, garlic, and apple, which were used for therapeutic purposes and taken as daily food. In this study, Bitter Gourd was the most preferred herbal product consumed by the T2DM patients.

\subsection{Major Themes}

This study categorised the findings obtained into three major themes: (a) Perception of conventional medicine, (b) Usage of conventional medicine, and (c) Intention to discontinue conventional medicine. The details of the themes are explained below: 
Table 1. Major themes and subthemes

\begin{tabular}{lll}
\hline Theme & Subtheme \\
\hline & $\bullet$ & Perceived the side effects of conventional medicine \\
a) Perception of conventional medicine & - Personal experience \\
& - & - Influence from family and friends \\
& $\bullet$ & Complementary with herbal medicine \\
& $\bullet$ & Alteration in dosage and time of conventional medicine \\
b) Usage of conventional medicine & & - To complement herbal medicine intake \\
& & - Feeling fed-up with conventional medicine \\
& & - To reduce the risk of side effects \\
\hline & $\bullet$ & No intention at all \\
c) Intention to discontinue conventional medicine & $\bullet$ & Have intention to quit \\
& $\bullet$ & Have intention to quit and even trying to quit \\
\hline
\end{tabular}

a) Perception on conventional medicine

- Perceived the side effect of conventional medicine

Fear of the side effects formed a barrier to the informants towards continuing their conventional medicine consumption. It made them reliant on herbal medicine for managing their diabetes. In particular, medicine was generally known to consist of chemical compounds, resulting in the informants' belief that it would affect their body.

Because they must affect the body. The thing that I am worried about is the effect on the kidney. That's why I feel like I want to stop the medication.. Because the medication consists of chemical ingredients, so I believe that it will affect our body (Informant 1, Male, Malay, 62).

Besides, the interview sessions clearly revealed the manner in which family members and friends could influence the informants' perception of conventional medicine. They would receive much information on kidney problems, the side effects and chemicals in medicine, and dissatisfaction with conventional medicine. Such dissatisfaction occurred when their conventional medicine failed to produce the desired effect, which was further compounded by their family members and friends suggesting for the informants to complement it with herbal medicine. These reasons underlined one of the many that pushed them to consider herbal medicine as a treatment method.

There are some people who said that taking medication is not good for our body and the kidney especially. That's why I am scared.... My friends said sooner or later, the kidney will have problems due to the medication (Informant 3, Female, India, 66)

Yes, my friends suggest complementing conventional medicine with herbal medicine. I did not feel comfortable taking the medicine because of the chemical ingredients, I am worried about the side effects on my kidney (Informant 7, Male, Malay, 65).

Hmm, it doesn't affect me much but according to my sister who is also a diabetic patient, she is having a lot of side effects because of conventional medicine. She feels like there is no difference if she takes the conventional medicine and feels better to not consume it (Informant 25, Male, India, 61).

The fear of side effects was also from the informants' own experiences. Two informants reported that they experienced a negative effect when consuming the medication, whereby they were unable to tolerate it due to hypoglycaemia and felt unwell. This led them to take herbal medicine instead.

For gliclazide, I noticed that if I did not take proper food, I will experience hypoglycaemia (Informant 4, Female, Malay, 43).

I am not feeling well when I consume medication from the hospital. So, my friends that have experienced the same thing before have shown and suggested that I take herbal medicine and stop taking medication from the hospital. So, until now, I have taken herbal medicine (Informant 7, Male, Malay, 65). 


\section{- Perceived benefits of conventional medicine}

Despite their negative perceptions, some informants adequately perceived the benefits of conventional medicine, expressing their trust in its efficacy and considered it as important in managing diabetes. Several of them experienced a negative effect when skipping the doses of their medications, including high blood glucose reading, a collapse, and numbness of the finger.

If I didn't take conventional medicine, it would affect my blood sugar level, and the reading will increase higher (Informant 27, Male, Malay, 63)

I forget to take my medication and I feel like I will collapse. But it was a long time ago... And if I didn't take the medication, my hands will feel numb (Informant 9, Male, Malay, 67).

If I didn't take the medication, I experience the feeling of numbness (Informant 3, Female, India, 66).

Moreover, some of the informants felt that conventional medicine did not have any side effects. They believed that thinking too much could yield negative consequences, which showed that the mind could be a powerful tool to convince the body towards positive thinking.

So far, thanks to God, I don't have any side effects. For me, a kind of person that did not think much, I don't think there is a side effect. Maybe if we think too much, then the effect will come (Informant 5, Female, Malay, 47)

b) Usage of conventional medicine

\section{- Complementary with herbal medicine}

A majority of the informants complement their conventional medicine intake with herbal medicine as most believed that the herbs were an additional component only. They mentioned that conventional medicine was compulsory in treating diabetes, while others reported feeling the importance of incorporating both forms of medicine into diabetes management. However, they were also doubtful about the adverse effects and would consider to seek further information regarding such usage from the experts, doctors and herbal practitioners. This shows that healthcare providers play an important role in disseminating information regarding both types of medication as the patients put their trust into them unconditionally.

No, I never stop my medicine. Now, I never stop, I just have the additional medicine.......for me, the herbs can only help. To say that it can treat diabetes, I don't think so. But hospital medicine is a must because it helps our health (Informant 4, Female, Malay, 43).

We cannot avoid modern medication; there are pros and cons and we may need to balance it with herbal medicine because we cannot deny it due to their benefits. For me, if someone wants to take care of his health, he must consider both medicines. But I don't know if taking both of them will have adverse effects or not. So, we need to refer to experts such as doctors and herbal practitioners. Before we take the medicine, we need to get the information first (Informant 16, Female, Malay, 42)

Despite the combined use of medication, one informant perceived herbal medicine more effective than conventional medicine. Upon using herbal medicine, he found that it was beneficial and yielded quick results compared to conventional medicine. However, he respected the fact that conventional medicine still played a role in the process.

I did not say government medication is not good, but for me when I take this herbal drink, I wake up in the morning feeling fresh and my body feels better...I did not say we cannot take the medication from the clinic but it takes a longer time. For diabetic patients, when taking the medicine, the blood glucose level is around eight to ten. But with herbal medication, it is less than eight (Informant 22, Male, Malay, 42).

- Alteration in dosage and time of conventional medicine

This study found that most of the informants altered the dosage and time to consume conventional medicine. They stated that they did not comply with the doctor's advice and adjusted the medication by themselves, one of the reasons for such alteration was to complement the herbal medicine intake. Some of them expressed that they experienced herbal medicine effectively and it yielded a positive effect for them.

The doctor asked me to take the medicine two times per day; two in the morning and two in the evening. But Inever take the medicine in the evening. I only take the herbal drink. As I said, when I drink the herbal product, I feel better and comfortable. But because I already received the medication from the doctor, I still take it. But frankly speaking, I only take the medicine in the morning during the day. It has been almost 3 years that I skip my medication in the evening (Informant 19, Female, Malay, 55). 
The first theme discussed the perception of informants regarding the negative side effects of conventional medicine, which made some of them alter the medicine intake to reduce the risks. For example, one informant explained her plans on scheduling her medication to ensure the chemical risk can be reduced.

Yes, I alter the medication intake during the fasting month especially. I would take one tablet during suhur and after breaking the fast, I didn't take the medicine. Then, I will change the schedule alternately. I do it like that; for another month until today, if I eat once per day, then tomorrow I will not take it...because I want to reduce the chemical effects of the medicine (Informant 1, Male, Malay, 62).

Besides, some of the informants were particularly critical of the routine treatment of conventional medicine. Feeling fed-up and tired with the routine, this led them towards altering the dosage and time of medicine intake.

When I feel like I have taken a lot of medicine, I adjust the intake time...I feel so lazy to take it and feel bored. So, if I take the medicine in the morning, I skip in the evening... I feel bad when I need to take a lot of medicines (Informant 3, Female, India, 66).

c) Intention to discontinue conventional medicine

The third and last theme discussed the intention to discontinue conventional medicine intake in managing diabetes. This theme consisted of three subthemes, including no intention at all, have the intention to quit, and have the intention to quit and are even trying to quit.

\section{- No intention at all}

Almost half of the informants did not have any intention to quit as they fully perceived the benefits and effectiveness of conventional medicine, which was discussed per the first theme. Their confidence and trust in conventional medicine following its provision by the government had become a strong reason for them to continue it. Moreover, they believed that external medicines might have side effects, in addition to the information relayed by diabetes experts to continue taking conventional medicine, despite also taking herbal medicine as an additional regiment.

I don't feel scared because the medicine is from the government, so I am confident...Yes, 100\% confident. If the medicine from outside, it might have an effect on us (Informant 6, Female, Chinese, 66).

I don't have the intention to quit. Because I meet everyone that said although herbal medicine has been suggested to treat diabetes, we cannot stop the medication from hospital. We need to continue. There are diabetes experts who said to never stop hospital medicine although we take alternative medicine; lessening the intake is okay but to never skip it (Informant 23, Male, Malay, 69).

One of the informants mentioned that T2DM patients must take conventional medicine continuously and consistently, as he believed that diabetes could not be cured.

Cannot. You cannot. Don't stop. Cannot stop because diabetes cannot be cured (Informant 28, Male, Chinese, 75).

- Have the intention to quit

Several informants reported that they had the intention to discontinue conventional medicine. This was due to various reasons, including their negative past experiences of feeling weak after taking the medication and their belief in the effectiveness of herbal medicine. These reasons led these patients to access herbal medicine as an alternative treatment and to maintain this approach if it allowed them to manage their blood glucose reading independently.

Yes, I have the intention to quit. If I can, I do not want to take medication anymore.... One of the reasons is it will make my body weak. I want to try and stop and I was thinking that if I get this herbal leaf, I want to stop my medication. Because I am confident. But this is my opinion, I don't know about others (Informant 1, Male, Malay, 62).

Yes, I want to stop conventional medicine. If herbal medicine can help why not? If I practice the herbal medicine and the blood sugar readings are okay, it is better that I stop the conventional medicine. I am planning to try for two weeks and discontinue the medication, I want to practice herbal medicine in the morning and night. If the blood sugar reading is good, I think I will stop my medication. But if the reading is high, I need to take it back (Informant 13, Female, Malay, 50).

- Have the intention to quit and even trying to quit

Notably, one informant did not just have the intention; he expressed his desire for the action by trying to quit conventional medicine a few times. However, the unimpressive results of blood sugar level obtained made him 
continue taking conventional medicine in managing his diabetes regardless.

I want to stop taking medication. I quit before...I want to test my blood glucose level. Yes, it was increased. I have tried to quit two to three times (Informant 11, Male, Malay, 52).

\section{Discussion}

This study explored the usage and perception of T2DM patients on conventional medicine and the manner in which it was used with herbal medicine in managing diabetes, namely those hailing from four government clinics in Negeri Sembilan, Malaysia. It was found that almost all of the patients used metformin for treating their disease, while half of them consumed it together with insulin. According to Wulffele et al., (2002), metformin is the commonest oral medication utilised to control diabetes and its function is to improve the patient's glycaemic control, especially in those with poorly controlled T2DM. However, a high number of T2DM patients are unable to attain a tight glycaemic control merely by using oral agents; thus, they require insulin either as a single agent or as an addition to the oral treatment. Moreover, Miller et al., (2009) have mentioned that T2DM is a chronic and progressive disease, which oftentimes requires two or more medications in order to achieve the ideal glucose control. This is important to prevent any cardiovascular complications (Derosa \& Sibilla, 2007).

Furthermore, this study identified Momordica Charantia (bitter gourd) as the most preferred herbal concoction for T2DM patients in treating their diabetes, besides Trigonella Foenum-Graecum (fenugreek), Orthosiphon Stamineus, and Clinacanthus Nutans (Sabah Snake Grass). All of the herbs consumed by the patients in this study have also been used by other T2DM patients worldwide (Mohamed et al., 2016). In 2013, Ghorbani conducted a systematic review to identify the herbs that could help in managing diabetes and supported by clinical studies. He found that the bitter gourd was one of the herbs that could reduce the blood glucose level, as well as other herbs such as Nigella sativa (black seed), Trigonella Foenum-Graecum (fenugreek), and Ociumum Sanctum (holy basil) got treating diabetes. Therefore, he suggested for physicians to depend on such herbs as complementary therapeutic options at minimum, alongside conventional medicine in order to enhance diabetic patient management.

Most of the patients perceived the negative side effects of conventional medicine, which led them to consider herbal medicine as a complementary alternative in managing their diabetes. This is a similar finding to those of other studies (Vickers et al., 2006; Hasan et al., 2011; Wachtel-Galor \& Benzie, 2011; Ali \& Mahfouz, 2014; Welz et al., 2018). Moreover, Derosa and Sibilla (2007) have discussed the fear of adverse effects further, which is usually the cause of discontinued conventional medicine intake, especially for those under multiple therapies.

Similarly, this study underlined the manner in which patients' perception of conventional medicine was influenced by the opinion of others such as family members and friends and the results them observed when consuming the medication. This is consistent with the findings from a previous study involving diabetic patients, namely the qualitative study conducted in Eastern Uganda by Rutebemberwa et al., (2013). The work has reported that the influence of family members and friends has thus contributed to the use of traditional medicine. Meanwhile, Welz et al., (2018) have also identified the factor of family members as one of the crucial sources of information regarding herbal medicine. Therefore, the outcomes of this study revealed that the influence of other people played a big role in the decision-making processes and health-based behaviours among diabetes patients.

In terms of medicine usage, this study reported that conventional medicine was used together with herbal medicine in which most informants believed that the herbs were merely additional inclusion in managing diabetes. According to Peter and Srinivasan (1997), natural herbal plants are used in treating diabetes as they are easily accessible and do not require laborious pharmaceutical synthesis processes. Besides, patients prefer consuming them due to high convenience such as no dietary restrictions required unlike when taking conventional medicine (Rutebemberwa et al., 2013). Accordingly, Bahall and Edwards (2015) have revealed that those who consume both types of medicine in combination feel that it is necessary and are not keen on experiencing the risks of declining conventional medicine.

In contrast, Neustadt (2006) and Bent (2008) have noted that the consumption of herbal and conventional medicines concomitantly may present adverse effects and cause serious health problems. Egede (2004) has also raised concerns about the risk of drug interaction and the possibility of the combination potentially worsening patient's glycaemic control or creating additional complications. Similarly, Ibrahim (2006) has stated that concurrent use of conventional and herbal medicines can lead to potential negative reactions or contraindications. Another scholar (Toklu, 2016) has also stated a few problems associated with the use of these herbal products, which include herb-drug interaction, improper labelling, contamination, unknown composition, the lack of standardisation for the active component dosage, and inappropriate dosing and pharmaceutical form. 
Furthermore, Chang et al., (2013) have indicated the need for healthcare professionals to acknowledge herbal medicine usage as the patients do not use herbal medicine in place of conventional medicine, but rather to complement it. Therefore, understanding patient usage for both types of medicine may be beneficial in order to minimise the risks of potential interaction. Besides, a standardised guideline promoting safe practice should be developed to understand patient's routine consumption with regard to natural product usage, as well as their individual plans for integrating natural products and conventional medicine (Chang et al., 2013).

According to Ibrahim (2006), healthcare professionals, especially pharmacists, should be trained and educated on the knowledge, indication, dosage, dose form, pharmacology, clinical controlled trials, potential adverse effects, and contraindications of herbal medicine. Such education regarding its advantages and safety needs to be included in the pharmaceutical curriculum across all local universities. However, Chang et al., (2013) have also admitted that the limited evidence-based research regarding natural product efficacy in laboratories and clinical practice both is still a barrier preventing healthcare professional to conduct informative education programmes.

Another key finding obtained in this study was the issue of dosage and consumption timing alterations made by patients for their prescribed conventional medicine. Most of the patients altered their regiment to complement their herbal medicine intake. This parallels the findings noted by Chang et al., (2013), whereby the patients consume the natural products either at a different timing compared to conventional medicine or self-lowering their prescribed dosage. Such alterations happened as they believed the natural products were safe for consumption and caused no adverse effects or interactions with their regimented conventional medicine.

This clashes the finding by McIntyre et al., (2015), who have stated that traditional herbal and complementary medicines can be problematic if used inaccurately. The act of self-preparation or self-medication by the patients may place themselves at risk of incorrect herb usage, such as consuming the wrong dosage or non-effectual preparations (e.g. non-standardised extracts), or selecting herbs capable of interacting with conventional medicine. This notion is in tandem with Chang et al., (2013), which has stated that self-administration of both types of medicine may endanger the patients. Besides, incorporating herbs of yet-to-be-proven clinical advantages may cause the patient to delay their treatment-seeking behaviours, thus causing complicated diabetes-related impacts and certain correlated disability and mortality outcomes (Rutebemberwa et al., 2013).

Beyond dosage alteration in order to complement herbal medicine, this study also attributed such action by patients to their dissatisfaction and unhappiness with daily consumption of conventional medicine and to reduce the risk of side effects. In particular, they found that it was challenging to consistently take their medications every day throughout their lives. According to Bukhsh et al., (2018), this is among the many key motivators of diabetic patients towards using herbal medicine. Similarly, Welz et al., (2018) have found that the issue of frustration and disappointment with conventional medicine prevails in their study, which may contextually lead to a decreased medication adherence among the patients. Moreover, Wroth and Pathman (2006) have further mentioned that patients' low adherence to their prescribed medication regiment may cause worse treatment outcomes and prognosis, as well as causing damage to the vital organs. These outcomes may thus result in a negative financial burden on each patient the society as a whole.

Last but not least, the findings of this study revealed that some of the patients were having the intention to discontinue their prescribed conventional medicine, while others were even trying to quit. This finding may be interpreted according to the fact that such patients may even discontinue conventional medicine in favour of complementary medicine (Bahall \& Edward, 2015). Therefore, it is vital for healthcare professionals to look deeper into the alteration issue and patient intention to discontinue conventional medicine, which may further impact the disease management.

\section{Conclusion}

This study successfully found that the findings yielded themes that were closely related to each other. The patients' perception of conventional medicine can influence their decision on both types of medicine usage (i.e. herbal and conventional), as well as their intention to quit from conventional medicine. In particular, the perception of negative side effects due to conventional medicine among these patients was highly influenced by the personal experiences and opinions of other people, such as their family members and friends. This influenced their intention to consume herbal medicine as a complementary option of conventional medicine.

Although all informants mentioned the consumption of conventional medicine in complement with herbal medicine, the act of altering their prescribed dosage and intake time in contrast to doctor's orders was the main concern. Here, an ineffective use of conventional medicine will lead to more critical effects and may cause serious health problems in the long-term. Moreover, such alteration may impact the patient's safety and expose them to 
unwanted side effects. Last but not least, the intention to stop taking the conventional medication was noted in some informants; this was the intention the patients had if herbal medicine could manage their diabetes on itself, which would need to be taken into account appropriately.

This study successfully gained deeper understanding into the areas surrounding the perception of T2DM patients regarding conventional medicine and its usage alongside herbal medicine. This will undoubtedly aid healthcare providers towards comprehending their current concomitant consumption in order to plan for future integration of both types of medicine. Additionally, understanding the patient's perception of conventional medicine and its concomitant use with herbal medicine will improve diabetes management as patients will be encouraged to undertake the appropriate and safe manner of usage.

\section{Acknowledgments}

We would like to thank the Director-General of Health and Deputy Director-General of Health (Research and Technical Support), Ministry of Health Malaysia for the permission to publish this paper.

\section{Competing Interests Statement}

The authors declare that there are no competing or potential conflicts of interest.

\section{References}

Ali, B., \& Mahfouz, M. S. (2014). Herbal Medicine Use among Patients with Type 2 Diabetes in North Sudan. Annual Research \& Review in Biology, 4(11), 1827-1838. https://doi.org/10.9734/arrb/2014/801

Appiah, B., Amponsah, I. K., Poudyal, A., \& Mensah, M. L. K. (2018). Identifying strengths and weaknesses of the integration of biomedical and herbal medicine units in Ghana using the WHO Health Systems Framework: a qualitative study. BMC Complementary and Alternative Medicine, 18(1). https://doi.org/10.1186/s12906-018-2334-2

Bahall, M., \& Edwards, M. (2015). Perceptions of complementary and alternative medicine among cardiac patients in South Trinidad: a qualitative study. BMC Complementary and Alternative Medicine, 15(1). https://doi.org/10.1186/s12906-015-0577-8

Baharom, N., Azhar, S., \& Rotina, A. B. (2016). Prevalence of complementary alternative medicine use among patients with type II diabetes in Negeri Sembilan, Malaysia. Medicine \& Health, 11(2), 257-266.

Bent S. (2008). Herbal medicine in the United States: review of efficacy, safety, and regulation: grand rounds at University of California, San Francisco Medical Center. Journal of general internal medicine, 23(6), 854-859. https://doi.org/10.1007/s11606-008-0632-y

Bukhsh, A., Gan, S. H., Goh, B.-H., \& Khan, T. M. (2018). Complementary and alternative medicine practices among type 2 diabetes patients in Pakistan: A qualitative insight. European Journal of Integrative Medicine, 23, 43-49. https://doi.org/10.1016/j.eujim.2018.09.003

Derosa, G., \& Sibilla, S. (2007). Optimizing combination treatment in the management of type 2 diabetes. Vascular health and risk management, 3(5), 665-671.

Egede, L. E. (2004). Complementary and alternative medicine use with diabetes. Geriatrics Times, 5, 54-59.

Ghorbani, A. (2013). Best herbs for managing diabetes: a review of clinical studies. Brazilian Journal of Pharmaceutical Sciences, 49(3), 413-422. https://doi.org/10.1590/s1984-82502013000300003

Hasan, S. S., Loon, W. C., Ahmadi, K., Ahmed, S. I., \& Bukhari, N. I. (2011). Reasons, perceived efficacy and factors associated with complementary and alternative medicine use among Malaysian patients with diabetes mellitus. The British Journal of Diabetes \& Vascular Disease, 11(2), 92-98. https://doi.org/10.1177/1474651410398367

Ibrahim, J. (2006). The Scientific Values of Malaysian Herbal Products. Jurnal Sains Kesihatan Malaysia, 4(1), $59-70$.

Institute for Public Health (IPH). (2015). National Health and Morbidity Survey 2015 (NHMS 2015) (Vol. II: Non-Communicable Diseases, Risk Factors \& Other Health Problems).

International Diabetes Federation. (2019). IDF Diabetes Atlas, 9th edn. Brussels, Belgium: 2019. Retrieved from http://www.diabetesatlas.org

Malviya, N., Jain, S., \& Malvia, S. (2010). Antidiabetic potential of medicinal plants. Acta Polomiac Pharma Drug Research, 67(2), 113-118. 
Mays, N., Ziebland, S., \& Pope, C. (2000). Qualitative research in health care: Assessing quality in qualitative research. $B M J, 320(7226), 50-52$.

McIntyre, E., Saliba, A. J., \& Moran, C. C. (2015). Herbal medicine use in adults who experience anxiety: A qualitative exploration. International journal of qualitative studies on health and well-being, 10, 29275. https://doi.org/10.3402/qhw.v10.29275

Miller, S. A., St Onge, E. L., \& Accardi, J. R. (2009). Sitagliptin as combination therapy in the treatment of type 2 diabetes mellitus. Diabetes, metabolic syndrome and obesity: targets and therapy, 2, 23-30. https://doi.org/10.2147/dmsott.s4068

Mohamed, H., Abdin, J., Lenjawi, B., Hamadani, Z., \& Schlogl, J. (2016). Use of Complementary and Alternative Medicine Among Patients with Type 2 Diabetes Mellitus in a Newly Developing Country: a Cross- Sectional Study in Qatar. International Journal of Clinical Research \& Trials, 1(2). https://doi.org/10.15344/2456-8007/2016/106

Mwangi, J., \& Gitonga, L. (2014). Perceptions and Use of Herbal Remedies among Patients with Diabetes Mellitus in Murang'a North District, Kenya. Open Journal of Clinical Diagnostics, 4(03), 152-172. https://doi.org/10.4236/ojcd.2014.4302

Neustadt, J. (2006). Herb-drug interactions: what clinicians need to know? Integrative Medicine, 5(1), 16-26

Ozkum, D., Ak1, O., \& Toklu, H. Z. (2013). Herbal medicine use among diabetes mellitus patients in Northern Cyprus. Journal of Medicinal Plant Research, 7(22), 1652-1664.

Remli, C., \& Chan S. C. (2003). Use of Complementary Medicine Amongst Diabetic Patients in a Public Primary Care Clinic in Ipoh. Medical Malaysia Journal, 58(5), 688-693.

Rojas, L. B., \& Gomes, M. B. (2013). Metformin: an old but still the best treatment for type 2 diabetes. Diabetology \& metabolic syndrome, 5(1), 6. https://doi.org/10.1186/1758-5996-5-6

Rutebemberwa, E., Lubega, M., Katureebe, S. K., Oundo, A., Kiweewa, F., \& Mukanga, D. (2013). Use of traditional medicine for the treatment of diabetes in Eastern Uganda: a qualitative exploration of reasons for choice. BMC international health and human rights, 13, 1. https://doi.org/10.1186/1472-698X-13-1

Toklu, H. Z. (2016). Pharmacovigilance of Herbal Medicine: Herbavigilance. Advances in Pharmacoepidemiology \& Drug Safety, 5(4). https://doi.org/10.4172/2167-1052.1000208

Vickers, K. A., Jolly, K. B., \& Greenfield, S. M. (2006). Herbal medicine: women's views, knowledge and interaction with doctors: a qualitative study. BMC complementary and alternative medicine, 6, 40. https://doi.org/10.1186/1472-6882-6-40

Vidya, K. R., \& Lohit, K. (2019). Complementary and alternative medicine use by diabetic patients in rural Bengaluru. International Journal of Community Medicine and Public Health, 6(11), 4863-4866.

Wachtel-Galor, S., \& Benzie, I. F. F. (2011). Herbal Medicine: An Introduction to Its History, Usage, Regulation, Current Trends, and Research Needs. In: Benzie IFF, Wachtel-Galor S, editors. Herbal Medicine: Biomolecular and Clinical Aspects (2nd ed.). Boca Raton (FL): CRC Press/ Taylor \& Francis; Chapter 1. Retrieved from https://www.ncbi.nlm.nih.gov/books/NBK92773/

Welz, A. N., Emberger-Klein, A., \& Menrad, K. (2018). Why people use herbal medicine: insights from a focus-group study in Germany. BMC Complementary and Alternative Medicine, 18(1). https://doi.org/10.1186/s12906-018-2160-6

World Health Organization (2013). Traditional medicine strategy 2014-2023.

World Health Organization. (2002). Traditional medicine strategy 2002-2005.

World Health Organization. (2005). National policy on traditional medicine and regulation of herbal medicines: report of a WHO global survey.

Wroth, T. H., \& Pathman, D. E. (2006). Primary Medication Adherence in a Rural Population: The Role of the Patient-Physician Relationship and Satisfaction with Care. The Journal of the American Board of Family Medicine, 19(5), 478-486. https://doi.org/10.3122/jabfm.19.5.478

Wulffele, M, G., Kooy, A., Lehert, P., Bets, D., Ogterop, J. C., Borger, B., Donker, A. J., \& Stehouwer, C. D. (2002). Combination of Insulin and Metformin in the Treatment of Type 2 Diabetes. Diabetes Care, 25(12), 2133-2140. https://doi.org/10.2337/diacare.25.12.2133 
Zayneb, A., Hayat, A., Bouchra, A., Youssef, H., \& Hanane, L. (2015). Herbal medicines use among diabetic patients in Oriental Morocco. Journal of Pharmacognosy and Phytotherapy, 7(2), 9. https://doi.org/10.5897/jpp2014.0338

\section{Copyrights}

Copyright for this article is retained by the author(s), with first publication rights granted to the journal.

This is an open-access article distributed under the terms and conditions of the Creative Commons Attribution license (http://creativecommons.org/licenses/by/4.0/). 